\title{
EFFICIENT WAVELET PACKET MODULATION FOR WIRELESS COMMUNICATION
}

\author{
Matthieu Gautier *॰, Marylin Arndt ${ }^{*}$ \\ ${ }^{*}$ France Telecom R\&D \\ 28, chemin du vieux chêne \\ 38243 Meylan - FRANCE
}

\author{
Joël Lienard $\diamond$ \\ ${ }^{\diamond}$ Laboratoire des Images et des Signaux \\ 961 rue de Houille Blanche \\ 38402 St Martin d'Heres - FRANCE
}

\begin{abstract}
Based on the good time-frequency localization of the pulse shaping, it is possible to build a multicarrier modulation that reduces both narrowband interferences and multipath channel interferences. In this paper, the given solution is based on wavelet theory: wavelet packet based multicarrier modulation is introduced and compared to the useful Orthogonal Frequency Domain Multiplexing (OFDM) modulation in a wireless environment with narrowband interferences and multipath channel interferences. Simulations show that the use of wavelet is more robust to narrowband interferences than the OFDM modulation. For multipath transmission, simulations show that the use of complex wavelet outperforms the use of real one and outperforms the OFDM modulation when the cyclic prefix technique is not used.
\end{abstract}

\section{INTRODUCTION}

In multicarrier modulation, the essential requirement on the elementary pulse shaping is the orthogonality with its timefrequency shifted versions. However, the propagation over the wireless channel leads to the loss of orthogonality, which could produce intersymbol (ISI) and interchannel (ICI) interferences. In a multipath environment with time dispersion, it has been proven that multicarrier transmission using OFDM modulation is very efficient [1]. By using a cyclic prefix symbol extension, ISI and ICI are completely cancelled. It results in an equalization by subcarriers which is extremely simple to implement. However, this symbol extension leads to a reduction of the bandwidth efficiency.

Therefore, to optimize the bandwidth efficiency, multicarrier transmission schemes without cyclic symbol extension are considered [2][3], they require a good time-frequency localization [4] of the elementary pulse. Based on the characteristics of the wireless channel, the characteristics of the pulse shaping could reduce both ICI, ISI and narrowband interference (NBI).

In order to design a multicarrier modulation with a significant time and frequency properties, a given solution is to use the wavelet theory. The application to filter bank and the extension to wavelet packet allow the construction of orthogonal bases used to modulate the data as a multicarrier system. A multicarrier modulation based on wavelet packet transform is called WPM (Wavelet Packet Modulation).
Wavelet theory applied to multicarrier modulation has been studied in previous works: it has been shown [5][6] that the WPM is efficient for wired transmission and that the "classical" WPM is not efficient in a time dispersive environment [7]. In this paper, WPM is applied effectively to wireless transmission, two different situations are considered: transmission with narrowband interference and transmission through time and frequency dispersive channel. Compared to [7], the major improvement of this paper is in the use of complex wavelet to reduce time and frequency dispersive channel interferences.

In the following, multicarrier transmission through wireless channel and the concept of wavelet packet modulation is first introduced in Sec. 2. Then transmissions through narrowband interference (Sec. 3) and through double dispersive channel (Sec. 4) are studied with both a discussion on the choice of the wavelet and simulation results. Finally, conclusions from simulations are drawn in Sec. 5.

\section{COMMUNICATION SYSTEM DESCRIPTION}

\subsection{Multicarrier Transmission}

Let $M$ be the number of channels in the multicarrier scheme. We consider a base of elementary signals $\left\{\psi_{m, n}(t), n \in\right.$ $\mathbb{Z}, m=0, \ldots, M-1\}$.

The transmitted symbols are denoted by $x_{m}[n]$. The index $n$ denotes the transmission time interval $[n T s ;(n+1) T s]\left(T_{s}\right.$ is the transmitted symbol duration) and $m$ the subcarrier number. The modulated signal results from a linear combination of the base functions weighted with the $x_{m}[n]$ :

$$
s(t)=\sum_{n=-\infty}^{+\infty} \sum_{m=0}^{M-1} x_{m}[n] \psi_{m, n}(t) .
$$

In the case of a non-selective fading channel and orthonormal functions, the demodulation symbols $y_{m}[n]$ are:

$$
y_{m}[n]=x_{m}[n] h_{m n}+b_{m}[n]
$$

where $h_{m n}$ and $b_{m}[n]$ are the resulting channel attenuation factor and noise for subcarrier $m$ and time symbol $n$ respectively. (2) leads to an equalizer composed of a single tap per subcarrier.

The OFDM modulation [1] uses a rectangular pulse shaping of duration $T_{s}$ and the orthogonality is attained for a carrier 
spacing of $1 / T_{s}$. By noting $\Pi_{0}^{T_{s}}(t)=\left\{\begin{array}{ll}1 & \text { if } 0 \leq t<T_{s} \\ 0 & \text { else }\end{array}\right.$, the rectangular function, $\psi_{m, n}(t)$ is then expressed by:

$$
\psi_{m, n}(t)=e^{j 2 \pi \frac{t}{T_{s}}} \Pi_{0}^{T_{s}}\left(t-n T_{s}\right) .
$$

A cyclic prefix of duration $\Delta_{C P}$ is then inserted between two OFDM symbols.

\subsection{Wireless transmission}

In this study, two interferences of wireless communication are considered and presented in the following.

\subsubsection{Narrowband interferences}

OFDM based Wireless Local-Area-Networks encounter interferences from Bluetooth devices. In this case, a narrowband interference (NBI) could model these interferences.

In this study, the narrowband interference is modelled by a sinusoidal wave [8] which interferes the data transmission. The interfering signal $j[n]$ is a sinusoid parameterised by its frequency $\nu_{j}$ and its power $P_{j}=10 \log \left(A_{j}^{2}\right)$ with $A_{j}$ the level of the sinusoid. The received signal $r[n]$ is the sum of the transmitted signal $s[n]$ with the interfering signal:

$$
r[n]=s[n]+j[n] \text { with } j[n]=A_{j} e^{j 2 \pi \nu_{j} n} .
$$

We note that the transmission is considered without another interference.

\subsubsection{Time and frequency dispersive channels}

The (complex) baseband double dispersive channel can be modelled by a random process in both time and frequency. The largest delay $\tau_{L}$ produced by the channel is called the multipath spread and the largest Doppler shift $f_{d}$ is called the Doppler spread. This effect of time dispersion is characterized in the frequency domain by the coherence bandwidth $B_{c}$ with $B_{c} \propto 1 / \tau_{L}$. The effect of frequency dispersion is characterized in the time domain by the coherence time $T_{c}$ with $T_{c} \propto 1 / f_{d}$.

\subsection{Wavelet packet modulation}

\subsubsection{Wavelet packet transform and filterbank}

The concept of wavelet transform has been extended by establishing the theory for libraries of orthonormal bases which were obtained by filling out the binary tree to some uniform depth as shown in Fig. 1. This decomposition allows a uniform analysis of the spectrum. The obtained functions are wavelet packets, which are recursively defined by:

$$
\begin{aligned}
p^{2 m}(t) & =\sqrt{2} \sum_{k} h_{k} p^{m}(2 t-n), \\
p^{2 m+1}(t) & =\sqrt{2} \sum_{k} g_{k} p^{m}(2 t-n) .
\end{aligned}
$$

$h_{n}$ and $g_{n}$ are a quadrature mirror filter (QMF) pair. $h_{n}$ is a lowpass filter while $g_{n}$ is a highpass filter. They are connected by the relation $g_{n}=(-1)^{n} h_{1-n}$.

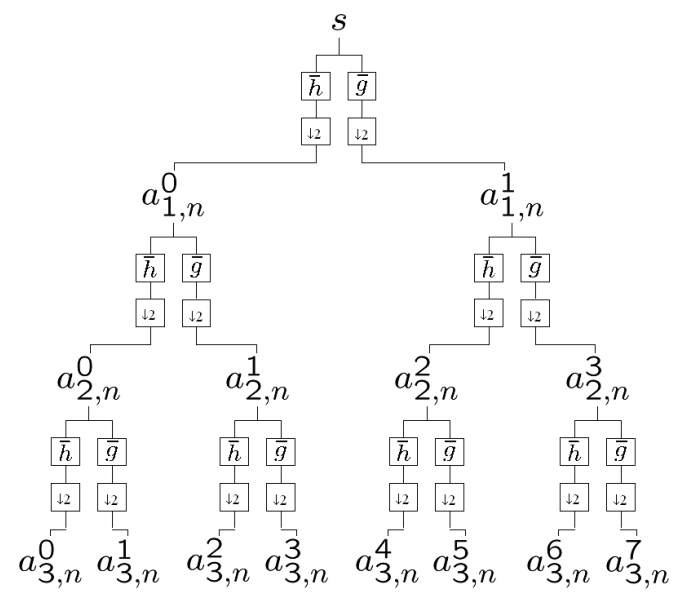

Fig. 1. Uniform wavelet packet decomposition.

Definition 1 A wavelet packet base of $L^{2}(\mathbb{R})$ is all orthonormal bases chosen among the functions:

$$
\left\{p_{l, n}^{m}(t)=2^{\frac{l}{2}} p^{m}\left(2^{l} t-n\right),(l, n) \in(\mathbb{Z}, \mathbb{Z}), m \in \mathbb{N}\right\} .
$$

Therefore, any function $f(t)$ of $L^{2}(\mathbb{R})$ can be decomposed on the base $\left\{p_{l, n}^{m}(t),(l, n) \in(\mathbb{Z}, \mathbb{Z})\right\}$ :

$$
s(t)=\sum_{l, n} a_{l, n}^{m} p_{l, n}^{m}(t)
$$

All these coefficients $a_{l, n}^{m}$ constitute the DWPT (Discrete Wavelet Packet Transform) of $s(t)$ and the inverse transform is called IDWPT (Inverse Discrete Wavelet Packet Transform).

\subsubsection{Wavelet packet multicarrier modulation}

From (8), we can see that any function $s(t)$ of $L^{2}(\mathbb{R})$ can be expressed as the sum of weighted wavelet packets. In communication systems, this means that a signal can be seen as the sum of modulated wavelet packets, which gives the idea of wavelet packet modulation [5]: the transmitter transforms the symbols from the wavelet domain to the time domain with an IDWPT and the receiver transforms the received signal from the time domain to the wavelet domain with a DWPT.

By choosing $M=2^{l}, \psi_{m, n}(t)=p_{l, n}^{m}(t)$ and $y_{m}[n]=a_{l, n}^{m}$, the tree shown in Fig. 1 represents an example of WPM demodulation for a $M=8$ subcarriers system. The orthogonality of wavelet packets gives a perfect reconstruction system for an ideal transmission without interference.

\section{NARROWBAND INTERFERENCES}

\subsection{Choice of the wavelet}

Firstly, the NBI's influence is studied for monocarrier transmission. The aim is to defined how the NBI interferes the modulated pulse shaping to determine its parameters.

The modulation consists in giving a waveform to the transmitted symbols around a chosen frequency $\nu_{m}$. For OFDM 
modulation, the complex exponential limited on the symbol duration $T_{s}$ is used:

$$
\psi(t)=e^{i 2 \pi \nu_{m} t} \Pi_{0}^{T_{s}}(t)
$$

We note $\hat{X}(\nu)$ and $X(\nu)$ the Fourier transforms of the received and transmitted symbols repectively, and $\Psi(\nu)$ the Fourier transforms of $\psi(t)$. It's simple to determine that for a sinusoidal NBI, the received symbols spectrum is:

$$
\hat{X}(\nu)=X(\nu)+A_{j} \Psi\left(\nu_{j}\right) .
$$

The spectrum $\hat{X}(\nu)$ of the received symbols is decomposed into two terms : a useful part corresponding to the spectrum $X(\nu)$ of the transmitted symbols and a part resulting from interferences. We can notice that NBI are contained in the term $\Psi\left(\nu_{j}\right)$ which is the value of the pulse shaping spectrum at the interfering frequency.

If the interfering frequency $\nu_{j}$ is equal to $\nu_{m}$, the transmission is, in an obvious way, very disturbed even impossible for a certain NBI's power $P_{j}$. However, when the interfering frequency $\nu_{j}$ is different to $\nu_{m}$, the transmission is also disturbed by the NBI. Indeed, the interfering signal will affect sidelobes of the pulse shaping spectrum. The more powerful the sidelobes are, the more disturbed the transmission will be.

In the case of rectangular pulse shaping used to modulate data, the spectrum is a cardinal sine. The first sidelobe is only $13 \mathrm{~dB}$ attenuated from the mainlobe.

In order to reduce the transmission sensitivity with reference to narrowband interference, we have to build a modulated pulse shaping with lower sidelobes than time-limited exponential waveform.

WPM allows the building of pulse shaping with very low sidelobes power. However, the attenuation depends on the choice and the complexity of the wavelet.

An example of a wavelet is shown in Fig. 2 by its power spectral density. The wavelet shown is the Daubechies wavelet with 12 coefficients. Daubechies wavelets are choosen because they are orthornormal wavelets with compact support. Daubechies wavelets are parameterized by the number of coefficients $L$ of filter $h_{n}$. Coefficients $h_{n}$ have been published by I. Daubechies in [9].

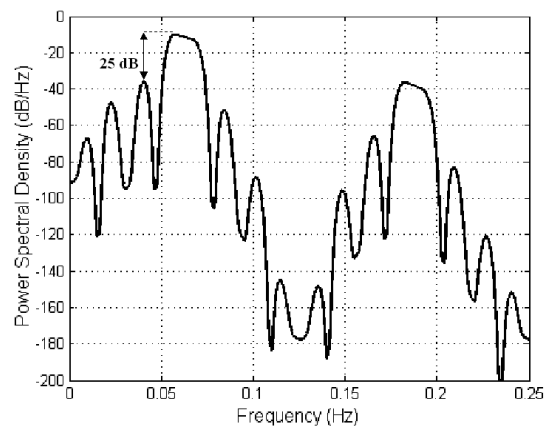

Fig. 2. Power Spectral Density of the Daubechies $(\mathrm{L}=12)$ wavelet.
The PSD of this wavelet waveform shows that sidelobes are at least $25 \mathrm{~dB}$ attenuated compared with the main lobe, whereas the attenuation is $13 \mathrm{~dB}$ for the time-limited exponential waveform.

So, the use of the wavelet allows the construction of a waveform with low sidelobes that should be more resistant to a NBI signal.

\subsection{Simulation results}

\subsubsection{Influence of narrowband interference on monocarrier transmission}

To test the sensitivity of the OFDM and WPM pulse shaping to the sinusoidal NBI, the system is initially reduced to the use of only one subcarrier. For the WPM, Daubechies wavelets with $\mathrm{L}=12$ and $\mathrm{L}=40$ coefficients are used.

The 4-QAM symbols are transmitted on subcarrier $\nu_{m}=$ 0.0625 . All the frequencies in this paper are normalized by the bandwidth $B$ required for the transmission $\left(\nu=\frac{f}{B}\right.$ with $f$ the real frequency).

The Mean Square Error (MSE) between transmitted and received symbols is calculated for a NBI with a frequency ranging from 0 to 0.2 and a power of $10 \mathrm{~dB}$. Results are shown in Fig. 3.

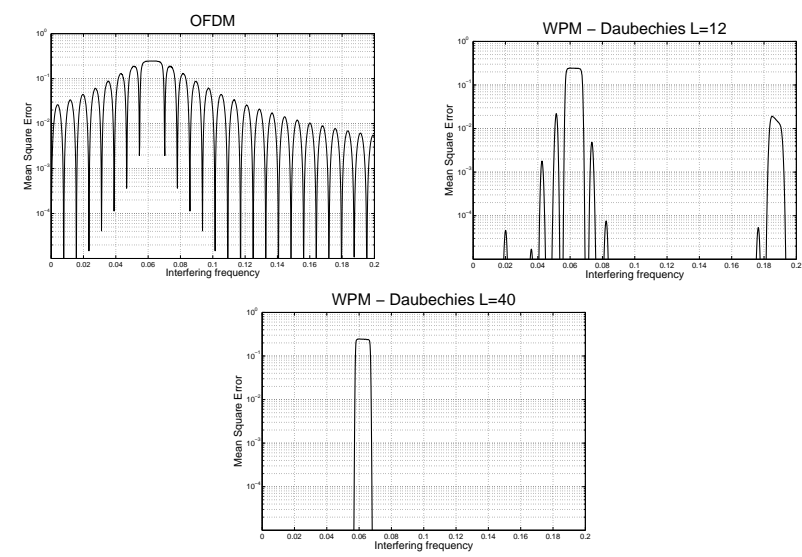

Fig. 3. Mean Square Error in the presence of a $10 \mathrm{~dB}$ power NBI as a function of interfering frequency for OFDM, WPM (Daubechies L=12) and WPM (Daubechies L=40) waveform.

By varying the interfering frequency, results show that errors are weaker for wavelets than for OFDM modulation. At the carrier's sidelobes, the effect of the interfering is completely cancelled for the WPM pulse shaping. The WPM pulse shaping is thus less sensitive to the NBI than the OFDM carrier. We can note the significant improvement due to the use of the Daubechies wavelet with $\mathrm{L}=40$ coefficients.

\subsubsection{WPM performances in presence of narrowband inter- ference}

Let's use a total system of multicarrier transmission. OFDM modulation and WPM are compared with 4-QAM symbols transmitted on $M=128$ carriers. Wavelets used for the 
WPM are Daubechies wavelets with $\mathrm{L}=12$ and $\mathrm{L}=40$ coefficients.

The used NBI has a variable frequency and measurements are made for a constant power of $10 \mathrm{~dB}, 20 \mathrm{~dB}$ and $30 \mathrm{~dB}$. Results in Binary Error Rate are shown in Fig. 4.
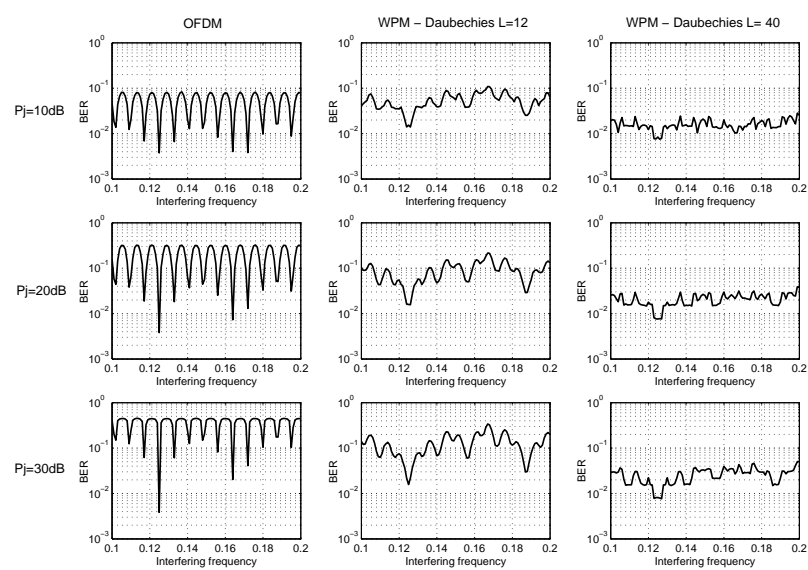

Fig. 4. Bit Error Rate as a function of interfering frequency for different power and for OFDM, WPM (Daubechies $\mathrm{L}=12$ ) and WPM (Daubechies $\mathrm{L}=40$ ) modulation.

Let's observe the number of errors made according to the interfering frequency, results show that WPM transmission are less sensitive to NBI than OFDM transmission. The performances of the WPM are more significant with the increase of the NBI's power.

We also note that the BER varies less according to the interfering frequency. Because of their construction, each WPM subcarrier is different, this explains the irregularities of the curves of BER.

\section{TIME AND FREQUENCY DISPERSIVE CHANNEL}

\subsection{Choice of the wavelet}

Wavelet theory offers a lot of orthogonal pulse shaping with good time-frequency properties. In this study, we will use the Daubechies wavelets. The choice of Daubechies wavelet and the use of complex wavelet is discussed in the following.

\subsubsection{Time-frequency Localization}

In multicarrier transmission over dispersive channels, the interference can be reduced [4] when the signal energy of a the pulse shaping is very concentrated around its center. This is measured by the frequency dispersion $\Delta F$ and the time dispersion $\Delta T$ of the pulse shaping.

Definition 2 We make use of the dispersion characteristics of the base functions and we postulate for ISI- and ICI-free transmission:

$$
\Delta T<<T_{c} \text { and } \Delta F<<B_{c} .
$$

If these conditions are satisfied, the channel can be considered as frequency non-selective and slowly fading for each carrier. Then, the transmission verifies (2) and a single tap equalizer per subcarrier can be used.

Table 1 gives the time and frequency dispersions for several Daubechies wavelets used by WPM and for the rectangular pulse used in OFDM modulation.

Table 1. Time-frequency Localization for different waveform.

\begin{tabular}{c|c|c} 
Waveform & $\Delta T$ & $\Delta F$ \\
\hline OFDM & 130.98 & 0.083 \\
Daubechies $(\mathrm{L}=12)$ & 249.3 & 0.068 \\
Daubechies $(\mathrm{L}=40)$ & 560.3 & 0.026
\end{tabular}

Daubechies wavelets are better localized in frequency than the rectangular waveform. However, they are worse localized in time. Thus, conclusions of time-frequency measurements are: according to (11), WPM should outperform OFDM modulation in a time dispersive channel and should not outperform OFDM modulation in a frequency dispersive channel.

\subsubsection{Drawback of common discrete wavelet}

Wavelets have good time-frequency localization. However, a major problem of the common discrete wavelet packet transform is its lack of shift invariance [10]. This means that on shifts of the input signal, the wavelet coefficients vary substantially. The signal information may even not be stationary in the subbands so that the energy distribution across the subbands may change. The shift dependence is illustrated

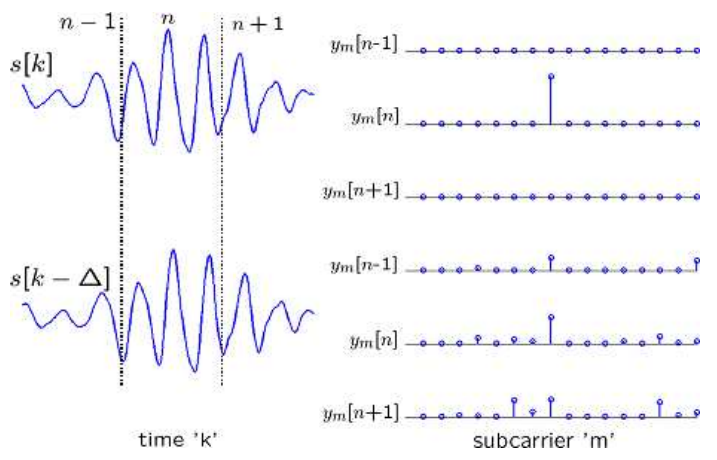

Fig. 5. shift sensitivity of the discrete wavelet packet transform.

in Fig. 5. For presentation purpose, a dilated Daubechies wavelet with $\mathrm{L}=12$ [9] is chosen as signal $s[k]$. Making a wavelet packet transform with itself, the result on the right is clearly a single non-zero coefficient resulting in a single subband with positive energy (only the coefficients absolute value is plotted). Now on a signal shift of $\Delta=7$ samples $s[k-\Delta]$, the other subbands of the wavelet packet transform also contain a significant portion of the signal energy.

This shows that the orthogonal discrete wavelet packet transform is highly sensible to the signal alignment relative to the subsampling points.

\subsubsection{Complex Wavelet}

To overcome the problem of shift dependence, one possible approach is to simply omit the subsampling causing the shift 
dependence. Techniques that omit or partially omit subsampling are also known as cycle spinning, oversampled filter banks or undecimated wavelet transforms. However, these transforms are redundant [11], which is not desirable in multicarrier modulation.

As an alternative, we used a non-redundant wavelet transform that achieves approximate shift invariance [12]. This transform yields to complex wavelet coefficients that modulate the data stream in the same way that for WPM.

We call the multicarrier modulation based on complex wavelet packet transform: Complex Wavelet packet Modulation (CWPM) [13]. In this study, simulations are limited to complex Daubechies wavelet.

\subsection{Simulation results}

Performances of the proposed system are compared to OFDM modulation with $\left(\Delta_{C P}=0.2 T_{s}\right)$ and without $\left(\Delta_{C P}=0\right)$ a cyclic prefix. All multicarrier systems are simulated with 4-QAM symbols modulated on $M=128$ subcarriers. Two systems using the wavelets are tested: a WPM system using real Daubechies wavelets, and one using complex Daubechies wavelets. Both have equalization with a single tap per subcarrier.

\subsubsection{Time dispersive channel}

The quality of the transmission depends on the multipath spread $\tau_{L}$ of the channel. In order to evaluate the influence of $\tau_{L}$ on the performance, a time invariant 2-paths channel is initially used. The received signal is expressed by $r[n]=s[n]+\alpha s\left[n-\tau_{L}\right]+b[n]$ with $\alpha$ the path's power, $\tau_{L}$ the path's delay and $b[n]$ an additive white gaussian noise define by the signal to noise power ratio $\frac{E_{b}}{N_{0}}$. Results in Bit Error Rate (BER) as a function of $\tau_{L}$ are shown in Fig. 6.

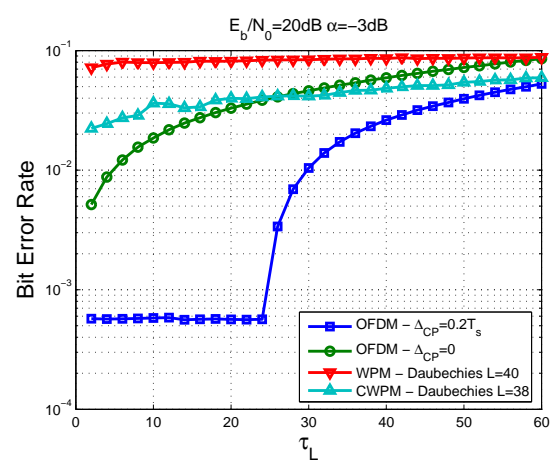

Fig. 6. Bite Error Rate (BER) as a function of $\tau_{L}$.

BER of WPM using complex waveform are lower than those of WPM using real waveform. The solution that consists in using complex wavelet instead of real one, is interesting. For almost the same number of coefficients, CWPM system outperforms WPM system.

Compared to OFDM modulation, results show that it is primarily the use of a cyclic prefix that gives to OFDM modulation interesting results in term of BER. WPM outperforms OFDM modulation only when the cyclic prefix is not the solution used.

\subsubsection{Frequency dispersive channel}

For a frequency dispersive transmission, the channel is now composed of a single time-varying path $\alpha$. The variation of the coefficient is parameterized by the maximum Doppler frequency $f_{d}$. In discrete time, the received signal is thus expressed by $r[n]=\alpha[n] s[n]$. The path has a unit power. Results in Mean Square Error (MSE) as a function of $f_{d}$ are shown in Fig. 7.

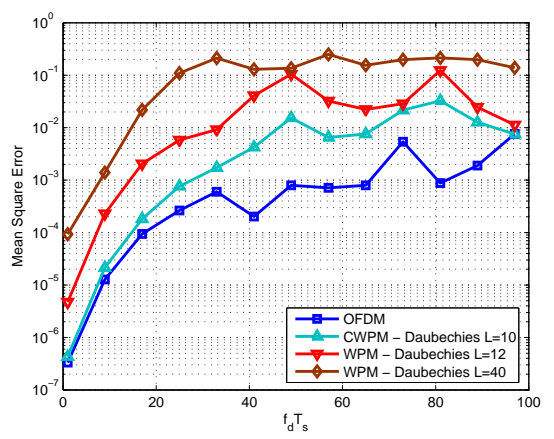

Fig. 7. Mean Square Error (MSE) as a function of $f_{d}$.

MSE of WPM system are higher than those of OFDM modulation. According to (11), the more significant the waveform frequency dispersion is, the more sensitive to the Doppler effect the modulation is. Thus, real Daubechies wavelet are more sensitive than the rectangular pulse shaping used by OFDM modulation.

We note that complex Daubechies wavelet is more robust than real Daubechies wavelet for an equivalent complexity.

\section{CONCLUSION}

In this paper, a multicarrier modulation system based on wavelet pulse shaping has been introduced.

In a NBI environment, WPM gives satisfactory results in Bit Error Rate compared with OFDM modulation. Our experiments have shown that WPM is less sensitive to a NBI, results are all the more significant as the NBI's power is high. For time and frequency dispersive channel, a new WPM system based on complex wavelet has been introduced. The use of complex wavelet outperforms the use of real one in both time and frequency dispersive channel transmission. Furthermore, the complex wavelet packets based multicarrier modulation has given satisfactory results compared to OFDM modulation. OFDM modulation shows limitations when the cyclic prefix is not the solution used. Our experiments have shown that the CWPM modulation is less sensitive in a time dispersive channel transmission. Yet, OFDM modulation is less sensitive than CWPM and WPM in a frequency dispersive channel transmission.

Moreover, WPM and OFDM modulation have nearly the same complexity according to the number of carriers [14] and wavelets allow flexibility in the system's design. The choice depends on a compromise between the desired performance and the system's complexity.

Future work will consist in developing the use of complex 
wavelet. Indeed, it has been proven that Meyer wavelet outperforms Daubechies wavelet for WPM using real wavelet. Thus, a solution to improve our results is to build a complex version of Meyer wavelet.

\section{REFERENCES}

[1] S. Weinstein and P. Ebert, "Data transmission by frequency-division multiplexing using the discrete fourier transform," 5, vol. 19, pp. 628-634, 1971.

[2] P. Siohan, C. Siclet, and N. Lacaille, "Analysis and design of OFDM/OQAM systems based on filterbank theory," 5, vol. 50, 2002.

[3] S. Trautmann and N.J. Fliege, "Perfect equalization for DMT systems without guard interval," 5, vol. 20, pp. 987-996, 2002.

[4] R. Haas and J.C. Belfiore, "A Time-Frequency Welllocalized Pulse for Multiple Carrier Transmission," Wireless Personal Communications, vol. 5, pp. 1-18, 1997.

[5] Alan R. Lindsey, "Wavelet Packet Modulation for Orthogonally Multiplexed Communication," 5, vol. 45, pp. 1336-1339, 1997.

[6] N. Erdol, F. Bao, and Z. Chen, "Wavelet Modulation A Prototype for Digital Communication Systems," Southcon/95. Conference Record, 168-171 1995.

[7] A. Jamin et P. Mähönen, "Wavelet Packet Modulation for Wireless Communication," Wireless Communications and Mobile Computing Journal, vol. 5, no. 2, March 2005.

[8] Marc Moeneclaey, Mark Van Bladel, and Hikmet Sari, "Sensitivity of multiple-access techniques to narrowband interference," IEEE Transactions on Communications, vol. 45, no. 3, March 2001.

[9] Ingrid Daubechies, "Orthonormal bases of compactly supported wavelets," Comm. Pure Appl. Math., vol. 41, 909-996 1988.

[10] Stephane Mallat, Une exploration des signaux en ondelettes, Ecole polytechnique edition, 2000.

[11] Ivan W. Selesnick, “The Double-Density Dual-Tree DWT," IEEE Transactions on Signal Processing, vol. 52, no. 5, pp. 1304-1315, Mai 2004.

[12] J-M Lina, "Complex daubechies wavelets: Filter design and applications," ISAAC Conference, June 1997.

[13] M. Gautier and J. Lienard, "Performances of complex wavelet packet based multicarrier transmission through double dispersive channel," NORSIG 06 IEEE Nordic Signal Processing Symposium (Iceland), June 2006.
[14] O. Rioul and P. Duhamel, Fast Algorithms for Wavelet Transform Computation, Chapter 2 of "Time-freq and Wavelets in Biomedical Engineering", 211-242, M. Akay Editor, IEEE Press edition, 1997. 